\title{
RECHERCHES SUR LA TENEUR EN CHLORE DU LAIT DE "OVIS ARIES ": SA SIGNIFICATION BIODYNAMIQUE ET BIOLOGIQUE DANS UN ÉCHANTILLON DE SUJETS ETHNIQUEMENT DÉFINIS (1)
}

\author{
par \\ Nino PINO et Luigi CHIOFALO \\ Institut de zootechnie générale de la Faculté de médecine vétérinaire \\ de l'Université de Messine
}

(Directeur : Prof. Dr. N. Pino)

Pratiquement, l'étude du rapport chlore-lactose (indice ou nombre de Koestler), et done la recherche du chlore tant par les méthodes habituelles à l'étable (chlorimétrie rapide) que par les méthodes plus scientifiques de laboratoire, est largement utilisée pour établir l'état de santé de la mamelle, duquel dépend évidemment la bonne qualité du lait pour les diverses destinations. Le fonctionnement troublé de la mamelle, altérant le rapport chlorelactose, conditionne comme on le sait une augmentation de la conductibilité électrique du lait avec rupture de l'équilibre entre ses principales constantes physico-chimiques. Comme on l'a observé dans de précédentes études, cet équilibre bio-physico-chimique contribue à caractériser le lait sous le rapport de son état naturel et de sa valeur nutritive potentielle.

La bibliographie démontre que la recherche du chlore a été effectuée avant tout sur le lait de vache. Etant donné l'importance de cela dans l'économie animale et étant donné aussi la carence de références bibliographiques dans ce secteur, on a prélevé sur quatre troupeaux de brebis Comisanes (brebis à tête rouge) élevées par le système de la semi-liberté dans la province de Messine, 94 sujets dont le lait a été soumis à l'analyse et les données, à l'élaboration biométrique.

De l'examen des résultats et de leur élaboration on peut relever :

a) Que la comparaison entre la moyenne arithmétique et son erreur standard (tableau I) établit que celle-ci est à considérer - et done sa signification sur le plan expérimental - identifiable avec la valeur vraie, théorique. Il en découle la possibilité de considérer un tel indice d'intensité comme valable pour les valeurs-chlore du

(1) Synthèse du rapport destiné au XIVe congrès de la Santé à Ferrare (Italie), mai 1967. 
lait d'ovins comparables, par les conditions d'alimentation, d'ambiance et d'hérédité, à ceux de l'échantillon. La véridicité établie de la valeur moyenne en met en valeur la fonction probante de valeur typique.

b) Que la valeur du sigma un peu élevée (tableau I) confirme ce qui a été noté dans la bibliographie au sujet de la variabilité

\section{TABLEAU 1}

DONNÉES SIGNALÉTIQUES DES VALEURS " CHLORE " DU LAIT DE " OVIS ARIES " $\mathrm{X}=$ moyenne; $\mathrm{D}=$ déviance $; \mathrm{V}=$ variance $; \sigma=$ déviation standard ; $\mathrm{Es}=$ erreur standard

$$
\begin{array}{cc}
\mathrm{X}= & \mathrm{mg} \cdot 98,219 \\
\mathrm{D}= & 16.534,969969 \\
\mathrm{~V}= & 177,795376 \\
\sigma= & 13,333 \\
\mathrm{Es}= & 1,3752
\end{array}
$$

Limites de sécurité de la moyenne

$$
\begin{aligned}
& X \pm t 0,05 . \text { Es } \\
& X \pm t 0,01
\end{aligned}
$$

$$
\begin{aligned}
& 98,219+1,99.1,3752=98,219+2,736648=100,955648 \\
& 98,219-1,99.1,3752=98,219-2,736648=95,482352 \\
& 98,219+2,638.1,3752=98,219+3,6277776=101,8467776 \\
& 98,219-2,638.1,3752=98,219-3,6277776=94,5912224
\end{aligned}
$$

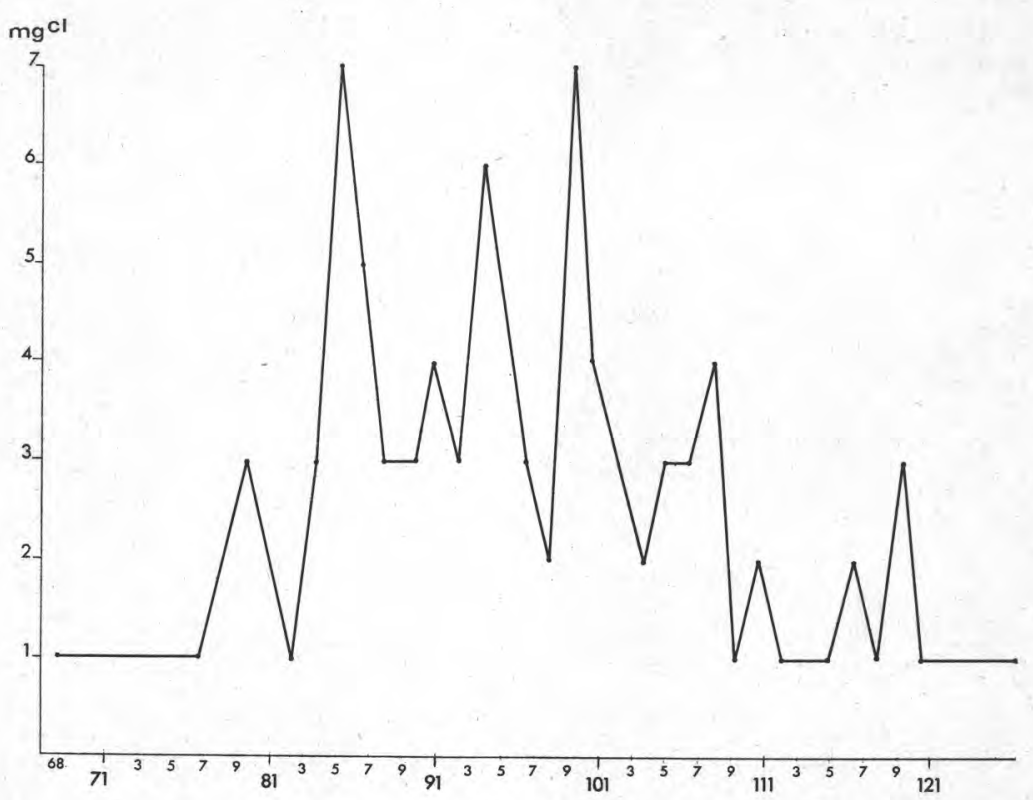

Fig. 1. 
accentuée de la teneur en chlore. Le diagramme relatif (fig. 1) rend plus évident l'évolution simple et collective du phénomène. La représentation graphique montre en effet une courbe plurimodale, expression d'hétérogénéité. Mais la concentration des fréquences et les pointes rapprochées offriraient un effondrement d'homogénéité et laisseraient déduire que l'évidente amplitude d'oscillation, portée des fluctuations individuelles, gravite entre des limites d'homogénéité de l'espèce.

Ces résultats et l'importance du chlore, que nous avons rappelée, dans l'économie animale ont conduit les auteurs à formuler des considérations au sujet de l'affirmation de Porcher et relatives à l'aspect biologique et biodynamique de ce constituant de lait ovin. 